\title{
THE ROLE OF HIV IN THE PATHOGENESIS OF ADULT-ONSET VERNAL KERATOCONJUNCTIVITIS: A DEMOGRAPHIC AND EPIDEMIOLOGICAL STUDY
}

Anine Kritzinger ( $\square$ aninekritzinger@gmail.com )

University of KwaZulu-Natal College of Health Sciences https://orcid.org/0000-0002-5244-5699

Anthony Grant Zaborowski

University of KwaZulu-Natal College of Health Sciences

Wilbert Sibanda

University of KwaZulu-Natal College of Health Sciences

Linda Visser

University of KwaZulu-Natal College of Health Sciences

\section{Research Article}

Keywords: Vernal keratoconjunctivitis, New-onset VKC Adult-onset VKC Ocular manifestations of HIV Allergy Immunocompromised

Posted Date: February 11th, 2019

DOI: https://doi.org/10.21203/rs.2.324/v1

License: (9) This work is licensed under a Creative Commons Attribution 4.0 International License. Read Full License 


\section{Abstract}

Background: Very few studies in the literature describe adult-onset vernal keratoconjunctivitis (VKC). HIV has many associated ocular pathologies and an association with VKC has not been described yet. The aim is to identify and describe patients who present with new-onset VKC after puberty, with no prior history of atopic diseases or allergies.

Methods: The study consisted of two parts: the first part was a prospective observational descriptive study of patients with adult-onset VKC, detailing the epidemiological and demographic characteristics of these patients, including their HIV status. The second was a case-control study to determine the relationship of a CD 4 count with adult-onset VKC in the setting of HIV. Patients were recruited between January 2016 and November 2017 from McCord Provincial Eye hospital, one of two large referral hospitals for the province of KwaZulu-Natal, South Africa. Patients presenting to the Eye clinic were screened at the Primary Eye Care Unit. Inclusion criteria were age 15 years and older with signs and symptoms of new-onset VKC. Exclusion criteria were a history of childhood atopic diseases, atopic keratoconjunctivitis and patients who refused HIV testing. Data collected included HIV status, CD4 count where appropriate, anti-nuclear antibodies and total serum immunoglobulin $\mathrm{E}$.

Results: 33 patients were included in this study; females $n=16$, males $n=17$. The mean age at presentation was $32.45 \pm 9.93$ years, $95 \% \mathrm{Cl}=28.94-35.97$. All of the patients were black Africans. One patient tested ANA positive. 51.5\% of patients had a raised IgE level. A total of 13 of 25 HIV positive patients $(52 \%)$ had a raised IgE. The proportion of HIV positive patients was statistically different from the HIV negative group, with Chi-squared $=21.866$, p-value $<0.0001 .72 \%$ of the HIV positive patients were grouped as immunodeficient according to their CD4 counts. An association was proven between severely immunodeficient patients and the risk of having VKC (chi-squared $=4.992, \mathrm{p}$-value $=0.0255$ ).

Conclusion: In this cohort a statistically significant association was found between adult-onset vernal keratoconjunctivitis and an HIV positive status. This association calls for more research on the subject, but could imply that patients presenting with adult-onset VKC should be offered an HIV test.

Key words: Vernal keratoconjunctivitis, New-onset VKC Adult-onset VKC Ocular manifestations of HIV Allergy Immunocompromised.

\section{Background}

Allergic conjunctivitis in HIV has been described in the literature [1]. Epidemiological studies about vernal keratoconjunctivitis (VKC) have noted that it may present as a new disease in young adults aged older than 15 years [2]. In other studies adult-onset VKC has been described as VKC with the onset in patients older than 20 years [3] and has been noted as very rare. Anecdotal evidence suggests that it is presenting itself regularly in our setting. 
The morbidity caused by immune-based hypersensitivity diseases might be a concern to the patient before the other manifestations of an immunocompromised status [4].

An epidemiological and demographic study on VKC done by Leonardi et al [2] found that adult-onset VKC presented as a new disease with an incidence of $0.06 / 100000$, compared to a mean incidence of $7.2 / 100000$ of VKC in children [2]. In our experience, patients present with the signs and symptoms of vernal keratoconjunctivitis in adulthood or after the onset of puberty, with no prior history of any atopic disease.

Research done on atopy and autoimmunity, showed that $35 \%$ of children tested positive for anti-nuclear antibodies (ANA) and this group of patients had the most significant clinical symptoms [5]. Another study investigating the pathogenesis of VKC found that $30.8 \%$ of patients tested positive for ANA [6].

Nebbioso et al. tested total serum immunoglobulin E ( $\lg E)$ in a study to investigate the role of individual variables in the pathogenesis of VKC. They found that $46.1 \%$ of patients had a total $\mathrm{IgE}>100 \mathrm{Ul} / \mathrm{ml}[6]$. Leonardi et al. found that total serum IgE testing done on VKC patients resulted in a wide range of results. Other studies demonstrated a high total serum IgE [7].

HIV has not been associated with VKC in previous studies.

For this study, a prospective review of patients with adult-onset VKC was done with the goal to identify and describe adult-onset VKC.

\section{Methods}

\section{Design and subjects}

This was a prospective, descriptive study.

The population sampled for this study was all patients presenting to the participating eye clinic at McCord Provincial Eye Hospital in Durban, South Africa. This is a public hospital with a catchment area that includes referrals from the rural coastal areas of KwaZulu-Natal, as well as inland areas, excluding Pietermaritzburg. The study population included any person aged 15 years and older with a diagnosis of new-onset vernal keratoconjunctivitis. Vernal keratoconjunctivitis is a disease that mostly subsides at or just before puberty [8]. Patients older than 15 years are grouped as adults and no longer part of the paediatric population [2]. The screening sister and doctor, as well as the sorting officer at the eye clinic, screened and sorted patients, identifying adult-onset VKC among patients. The sampling strategy was a consecutive sample that met the inclusion criteria. The sampling period was January 2016 to November 2017.

Patients who presented with a typical history, as well as signs and symptoms of vernal keratoconjunctivitis, were counselled regarding voluntary participation in this study. Each patient gave written informed consent, assisted by a translator where appropriate, to participate in this study, to 
provide blood samples and demographic as well as clinical data. Participants aged 15-18 years gave written assent to be a participant and a parent or guardian gave written informed consent. A data collection form, designed for use in this study and randomly numbered, was filled in by the consulting doctor.

The following information was collected: age, sex, race, general medical and atopic history, drug history including the use of antiretroviral medication and HIV status. KwaZulu-Natal is the province with the highest HIV prevalence (16.9\%) in South Africa [9].

Investigations done included ANA and total serum IgE. HIV testing was done if the patient's status was unknown and a CD4 count was done for HIV positive patients. Each patient's HIV status was treated as confidential by the investigator as well as the test counsellor.

All patients received the routine management and treatment for vernal keratoconjunctivitis.

The inclusion criteria were age 15 years and older, signs and symptoms of vernal keratoconjunctivitis, including: tearing, itching eyes, conjunctival papillae, limbal follicles, Trantas' dots, as well as any complications occurring secondary to vernal keratoconjunctivitis, such as a corneal shield ulcer.

The exclusion criteria were any patient younger than the age of 15 years, any patient with a history of childhood atopic diseases, such as allergic conjunctivitis, vernal keratoconjunctivitis, asthma, eczema, allergic rhinitis, and any patient with atopic keratoconjunctivitis. Patients who were unwilling to undergo HIV testing at the time of counselling were excluded from the study.

The HIV positive patients were grouped according to CD4 counts using the WHO system for immunological classification of an established HIV infection [10]. Patients were also grouped according to age. The total serum IgE levels were classified as normal or raised based on reference ranges according to age [11].

This study was approved by the Biomedical Research Ethics Committee (BREC) of the University of Kwazulu-Natal (BE519/14), and site permission was obtained from the medical manager of the participating hospital, McCord Provincial Eye Hospital.

\section{Statistical analysis}

Continuous variables were expressed as mean \pm standard deviation or medians, and these were compared using the Student's t-test. Proportions and categorical variables were compared using Pearson's chi-squared test or Fisher's exact test as appropriate. A linear correlation was assessed using Pearson's correlation coefficient (r).

All analyses were done using IBM SPSS version 25 (SPSS Inc. Released 2017. SPSS for Windows version 25. Armonk, NY: IBM Corp). The level of significance was set at $p<0.05$. 


\section{Results}

The study cohort included a total of 33 patients. The study consisted of 16 females, $(48.5 \%), 95 \% \mathrm{Cl}$ (32.5-64.8) and 17 males (51.5\%), 95\% Cl (35.2-67.5). All the demographic characteristics are shown in Table 1.

The youngest patient was 15 years old, and the oldest patient was 56 years old. The mean age of onset of VKC was 32.45 years $\pm 9.93,95 \% \mathrm{Cl}(28.94-35.97)$. The median age was 32 years (interquartile range $(I Q R)=12)$ and modal age of 26 years. The patients were divided into five groups according to their age (Table 1). At the time of diagnosis, more than $50 \%$ of the patients were older than 32 years, and $39.4 \%$ of patients were 30-40 years old. Females $(n=16)$ presented at an average age of $34.69 \pm 8.11$ years, $95 \% \mathrm{Cl}$ (30.36-39.01). The median age of presentation for females was 32.50 years (IQR $=7)$. The youngest female was 24 years, and the oldest was 56 years old. Males $(n=17)$ presented at an average age of $30.35 \pm 11.21$ years. The median age of males at presentation was 28 years $(I Q R=16)$. The youngest male was 15 years old, and the oldest male was 53 years old. The average age of presentation with VKC was higher in females ( $34.69 \pm 8.11$ years) than in males ( $30.35 \pm 11.21$ years), but this difference was not statistically significant at $95 \% \mathrm{Cl}, \mathrm{t}=-1.802 ; p$-value $=0.0763$.

All of the patients were black Africans.

Patients were tested for ANA and one patient out of 33 tested ANA positive.

All patients were tested for total serum IgE levels with an average IgE of $1862 \pm 2007.13$. $51.5 \%$ of patients had a raised IgE level, $42.4 \%$ had an IgE result that was within the normal range for their age and the rest, $6.1 \%$, did not have data (test results not available). In determining the mean, only those patients who had IgE values were included. 13 out of 25 HIV positive patients $(52 \%)$ had a raised IgE while four of the six HIV negative patients (66.7\%) had a raised serum IgE level.

HIV testing was done if a patient did not know their status and seven patients were HIV negative, this is $21.2 \%$ of the cohort, $95 \% \mathrm{Cl}(11-38) .26$ patients were HIV positive; this is $78.8 \%, 95 \% \mathrm{Cl}(62-89)$. The proportion of HIV positive patients was statistically different from the HIV negative group, Chi-squared = $21.866, p<0.0001$.

CD4 counts were done on all the HIV positive patients if their CD4 counts were unknown to them and grouped according to the WHO staging. The CD4 counts ranged between 33 and 1252 . The average CD4 count was $383.90 \pm 367.32$. The median for the CD4 count was $233(I Q R=467)$.

The risk of VKC was associated with any HIV associated immunodeficiency (chi-squared $=9.486$, $p=0.0021$ ), with a total of 25 patients of 33 testing HIV positive. In the group of HIV positive patients, 18 $(72 \%)$ of these patients were grouped as immunodeficient according to their CD4 counts. In this group, ten patients (40\%) had the lowest CD4 count (severely immunodeficient), and three patients (12\%) were mildly immunodeficient. An association was found between the severely immunodeficient group and the 
risk of VKC (chi-squared $=4.992, p$-value $=0.0255)$. There was a weak negative correlation between CD4 count and IgE (Pearson's $r=-0.2492)$ which was not statistically significant $(p=0.285)$.

There was no association found between sex and the risk of VKC, Chi-squared $=0.06$ and $p=0.8064$.

This study did not find a statistically significant association between HIV status and IgE levels ( $p$-value $=$ 0.485).

\section{Discussion}

Adult-onset VKC is relatively rare compared to childhood VKC [2] and as a result, a group of only 33 patients met the criteria for inclusion in this study. Numerous studies have been done on VKC in children, but very few studies have been conducted on VKC in adults. An example of a study is by Leonardi et al. (2013) where 49 patients with a VKC-like disease was found among 600 consecutive VKC patients and clinical data, demographic information and quality of life assessments were done. Immunologic data was collected and testing such as allergen sensitivity and tear cytokines were done. HIV status was not included in their data collection. Other extensive epidemiological studies have been done on VKC but do not mention VKC in adults [12].

Our study was done at a provincial hospital and patients may have been treated at their local clinics or hospitals for VKC, without a referral, possibly resulting in a lower number of patients seen at the provincial hospital.

VKC is typically a disease of males in childhood [8], but this study did not show a male predominance in adults, which may demonstrate a possible hormonal component in childhood VKC, as previously suggested in the literature [2]. However, this has not been proven yet and it calls for further study to determine the basis of this finding.

The incidence of HIV in KwaZulu-Natal is $2.3 \%$ and the prevalence is $16.9 \%$ [9]. In our study, $78.8 \%$ of patients in the cohort tested HIV positive and there was an association between VKC and HIV which was statistically significant. Therefore, it can be inferred that an HIV positive status is associated with a higher risk of VKC. The literature showed that some ocular manifestations of HIV in an immunocompromised patient could include atopic disease such as allergic conjunctivitis [1]. The small number of patients in our study could be due to the new Universal Test and Treat protocol [9] that South Africa implemented for HIV patients in 2016, whereby antiretroviral treatment is initiated immediately for all HIV positive patients, regardless of their CD4 count. This will leave fewer patients with a compromised immune system and manifestations of HIV.

The association between allergy and an HIV positive status is still poorly understood [1]. A correlation was seen between a lower CD4 count (clinically immunocompromised patients) and the risk of VKC. This may correlate with existing knowledge that ocular manifestations of HIV may be the presenting complaint while the patient may be otherwise well, and also that the frequency of ocular manifestations 
of HIV will increase as the patient's CD4 count decreases [13]. Other studies have noted an increased serum IgE level in HIV positive patients [14], but in this study, there was no statistically significant difference between the serum IgE level in HIV positive and HIV negative patients. This could be because patients were already on highly active antiretroviral therapy (HAART) when presenting to the Eye clinic. It has also been shown in the literature that VKC itself could be associated with a raised serum IgE level in at least $40 \%$ of patients [15] which could account for the raised serum IgE level in the HIV negative patients.

At the time of diagnosis, more than $50 \%$ of the patients were older than 32 years, and $39.4 \%$ of patients were 30-40 years old. The prevalence of HIV was highest among women aged 30-34 (36\%) and among men aged 35-39 (28.8\%) [16]. The HIV prevalence rate by sex and age in South Africa shows the highest rate in women aged 30-34 years and in men aged 35-39 years [16].

Several questions have been raised and need more investigation, such as the incidence and prevalence of adult-onset VKC, the role of HIV in the pathogenesis of VKC and the role of antiretroviral treatment in the onset and resolution of VKC. Further research is needed with a larger cohort of patients with adult-onset VKC. The monitoring of symptoms and the CD4 count could prove valuable.

This study is limited by the small number of patients.

\section{Conclusion}

In this cohort of patients, an association was found between adult-onset VKC and HIV. This study also found a statistically significant association between an immunocompromised status and the risk of VKC. Based on our findings, we could recommend that clinicians working in a setting with a high HIV prevalence rate consider offering HIV testing if patients present with new-onset VKC in adulthood, without any prior atopic history. This could prove to be significant if the patient falls into the high-risk age group for HIV.

\section{Abbreviations}

VKC: vernal keratoconjunctivitis

HIV: human immunodeficiency virus

ANA: antinuclear antibodies

IgE: immunoglobulin E

HAART: highly active antiretroviral therapy

\section{Declarations}




\section{Ethics approval and consent to participate}

This study was approved by the Biomedical Research Ethics Committee (BREC) of the University of Kwazulu-Natal (BE519/14), and site permission was obtained from the medical manager of the participating hospital, McCord Provincial Eye Hospital.

\section{Consent for publication}

Not applicable.

\section{Availability of data and material}

The datasets used and/or analysed during the current study are available from the corresponding author on reasonable request.

\section{Competing interests}

The authors declare that they have no competing interests.

\section{Funding}

Not applicable.

\section{Authors' contributions}

AK and AZ substantially contributed to conception and design. AK and WS significantly contributed to the interpretation of data and drafting the article, LV and AZ revised it critically for important intellectual content and approved the final version to be published.

\section{Acknowledgements}

Staff at McCord Provincial Eye Hospital

\section{References}

1. Haritha V KM, Srinivasulu Reddy Y, Sudhakar P. Allergic conjunctivitis as the initial ocular manifestation of HIV in young/middle age patients [20-40yrs]. International journal of science and research. 2015; 4(1):1577-1579.

2. Leonardi A, Busca F, Motterle L, Cavarzeran F, Fregona IA, Plebani M, et al. Case series of 406 vernal keratoconjunctivitis patients: a demographic and epidemiological study. Acta Ophthalmol Scand. 2006; 84(3):406-410.

3. Saboo US, Jain M, Reddy JC, Sangwan VS. Demographic and clinical profile of vernal keratoconjunctivitis at a tertiary eye care center in India. Indian J Ophthalmol. 2013; 61(9):486-489.

4. Marshall GD, Jr. AIDS, HIV-positive patients, and allergies. Allergy Asthma Proc. 1999; 20(5):301-304. 
5. Zicari AM, Nebbioso M, Lollobrigida V, Bardanzellu F, Celani C, Occasi F, et al. Vernal keratoconjunctivitis: atopy and autoimmunity. Eur Rev Med Pharmacol Sci. 2013; 17(10):1419-1423.

6. Nebbioso M, Zicari AM, Celani C, Lollobrigida V, Grenga R, Duse M. Pathogenesis of Vernal Keratoconjunctivitis and Associated Factors. Semin Ophthalmol. 2015; 30(5-6):340-344.

7. Bonini S, Coassin M, Aronni S, Lambiase A. Vernal keratoconjunctivitis. Eye (Lond). 2004; 18(4):345351.

8. Leonardi A. Management of vernal keratoconjunctivitis. Ophthalmol Ther. 2013; 2(2):73-88.

9. Universal test and treat "A Game Changer in HIV Prevention". 2016. http://www.kznonline.gov.za/hivaids/councils/Provincial-Councils-onAIDS/2016/Universal\%20Test\%20\&\%20Treat\%20Presentation.pdf. Accessed November 2017.

10. WHO case definitions of HIV for surveillance and revised clinical staging and immunological classification of HIV-related disease in adults and children 2017. http://www.who.int/hiv/pub/guidelines/WHO\%20HIV\%20Staging.pdf. Accessed November 2017.

11. Martins TB, Bandhauer ME, Bunker AM, Roberts WL, Hill HR. New childhood and adult reference intervals for total IgE. J Allergy Clin Immunol. 2014; 133(2):589-591.

12. Leonardi A, Lazzarini D, Motterle L, Bortolotti M, Deligianni V, Curnow SJ, et al. Vernal keratoconjunctivitis-like disease in adults. Am J Ophthalmol. 2013; 155(5):796-803.

13. Bekele S, Gelaw Y, Tessema F. Ocular manifestation of HIV/AIDS and correlation with CD4+ cells count among adult HIV/AIDS patients in Jimma town, Ethiopia: a cross sectional study. BMC ophthalmology. 2013; 13:20.

14. Bolad A, Ahmed I, Lutfi M. Reliability of IgE antibodies as a screening test for HIV infected Sudanese patients. FS J Pharm Res. 2012;1(4):73-74

15. Bozkurt B, Artac H, Arslan N, Gokturk B, Bozkurt MK, Reisli I, et al. Systemic atopy and immunoglobulin deficiency in Turkish patients with vernal keratoconjunctivitis. Ocul Immunol Inflamm. 2013; 21(1):28-33.

16. Kanabus A. HIV statistics South Africa. 2012. In:Information about tuberculosis. https://www.tbfacts.org/hiv-statistics-south-africa/. Accessed November 2017.

\section{Tables}

Table 1: Demographic Characteristics 


\begin{tabular}{|c|c|c|c|}
\hline Variable & $\mathbf{N}$ & $\%$ & $95 \% \mathrm{CI}$ \\
\hline \multicolumn{4}{|l|}{ Gender } \\
\hline Female & 16 & 48.48 & $33.5-64.8$ \\
\hline Male & 17 & 51.52 & $35.2-67.5$ \\
\hline Total & 33 & 100.0 & \\
\hline \multicolumn{4}{|l|}{ Age (Years) } \\
\hline$<20$ & 3 & 9.1 & 3.1-23.6 \\
\hline $20-30$ & 11 & 33.3 & $19.8-50.4$ \\
\hline$>30-40$ & 13 & 39.4 & $24.7-56.3$ \\
\hline$>40-50$ & 3 & 9.1 & 3.1-23.6 \\
\hline$>50$ & 3 & 9.1 & $3.1-23.6$ \\
\hline Total & 33 & 100.0 & \\
\hline \multicolumn{4}{|l|}{ Race } \\
\hline Black African & 33 & 100 & $89.6-100.0$ \\
\hline \multicolumn{4}{|l|}{ HIV Status } \\
\hline Negative & 7 & 21.2 & $10.7-37.8$ \\
\hline Positive & 26 & 78.8 & 62.3-89.3 \\
\hline Total & 33 & 100.0 & \\
\hline \multicolumn{4}{|l|}{ ANA } \\
\hline Negative & 30 & 90.9 & 76.4-96.9 \\
\hline Positive & 1 & 3.3 & $0.5-15.3$ \\
\hline Equivocal* & 1 & 3.3 & $0.5-15.3$ \\
\hline Not done & 1 & 3.3 & $0.5-15.3$ \\
\hline Total & 100 & 100 & \\
\hline \multicolumn{4}{|l|}{ CD4 Count } \\
\hline None & 7 & 21.2 & $10.7-37.8$ \\
\hline Mild & 3 & 9.1 & 3.1-23.6 \\
\hline Advanced & 5 & 15.2 & $6.7-30.9$ \\
\hline Severe & 10 & 30.3 & $17.4-47.3$ \\
\hline
\end{tabular}




\begin{tabular}{|l|l|l|l|} 
Not Applicable\# & 7 & 21.2 & $10.7-37.8$ \\
\hline Not done & 1 & 3.0 & $0.5-15.3$ \\
\hline Total & 33 & 100 & \\
\hline IgE & & & \\
\hline Normal & 14 & 42.4 & $27.2-59.2$ \\
\hline Raised & 17 & 51.5 & $35.2-67.5$ \\
\hline Not done & 2 & 6.1 & $1.7-19.6$ \\
\hline Total & 33 & 100 & \\
\hline
\end{tabular}

*Equivocal ANA result: fluorescence is borderline and needs further testing such as anti-nuclear factor \#Not applicable: pertaining to patients who are HIV negative 